\title{
Impact analysis of laminated and sandwich composites using a plate finite element with strain energy updating
}

\author{
U. Icardi \& L. Ferrero \\ DIASP - Politecnico di Torino, Italy
}

\begin{abstract}
This paper deals with finite element simulation of low velocity-low energy impacts on sandwich composites with laminated faces. A refined zig-zag model with a high-order piecewise representation of in-plane and transverse displacement components is used as a structural model in order to accurately simulate the effects of the transverse normal stress and strain. The goal is to develop a tool for improving the accuracy of conventional plate models, so as to enable the impact analysis of sandwich composites. A strain energy updating process is used for this purpose. As is customary, the Hertzian law and the Newmark implicit time integration scheme are used. The contact radius is computed within each load step by an iterative algorithm, which forces the impacted top surface to conform, in the least-squares sense, to the shape of the impactor. Then, the failure analysis is performed and the material properties of the failed areas reduced. Nonlinear strains of von Karman type are used because the transverse displacement can be quite large even when the plate deflection is small. Comparison with numerical and experimental results published in literature show the present model to be able to accurately predict the impact force and the damage it induces.
\end{abstract}

Keywords: low velocity impacts, induced damage, FEA, sandwich structures.

\section{Introduction}

Dynamic loading due to the impact of foreign objects represents a serious design concern for laminated and sandwich composites. Local damage appears as a visible permanent indentation in laminated composites for high velocity (or energy) impacts, while at low velocity it appears as global damage and mainly 
consists of invisible extensive internal delaminations. In sandwich composites, the damage occurs at low energies as invisible core crushing, while at energies exceeding a certain threshold, visible damage appears, followed by the complete tearing of the impacted face sheet. Despite the damage not being evident, the case of low velocity, low energy impacts is of great interest, because they are always responsible for relevant structural properties degradation; the transverse shear waves reflect off the edges several times while the contact load is still being applied, thus the lay-up, the constituent materials, the size and the boundary conditions strongly affect the response (see, e.g., Lin and Hoo Fatt [1]), while the strain-rate effects are negligible (see Suvorov and Dvorak [2]). This paper deals with finite element simulation of such case.

Having an inherent three-dimensional nature, low-velocity impact problems have been extensively investigated using conventional 3D displacement-based FE models; unfortunately, they require a large number of subdivisions across the thickness. To avoid such refined meshes, three-layer sandwich models have been used in several cases.

In addition, the so-called quasi-3D models, or partial layerwise models, have not found a massive application to impact studies, despite being effective without requiring an excessive mesh refinement, if appropriate post-processing techniques are applied. A recent application of these models to impact studies is presented in the paper by Palazotto et al. [3].

Refined zig-zag models with a high-order piecewise representation of inplane and transverse displacement components have been recently developed by the senior author [4-6] to conjugate accuracy and low computational costs. In this paper, an improved version of the strain energy updating and postprocessing procedures, an improved modelling of the contact force, an improved description of the transverse deformation and geometric nonlinearity are incorporated. The impact force and the damage predicted by the actual model are compared with those of experiments performed by the authors or published in literature, for a variety of sample cases.

\section{Structural model}

Laminated and sandwich composites need the local stresses occurring in their heterogeneous microstructure to be accurately accounted for in the region where damage rises. These contact conditions, which imply the transverse shear stresses $\sigma_{\mathrm{xz}}, \sigma_{\mathrm{yz}}$, the transverse normal stresses $\sigma_{\mathrm{zz}}$ and its gradient $\sigma_{\mathrm{zz}, \mathrm{z}}$ and the displacements (in absence of bonding and delamination damage) to be continuous at the interfaces, can be fulfilled in different ways. In a broad outline, they can be enforced as constraint conditions at the interfaces, assuming the displacements separately for each computational layer (discrete-layer models), or postulating an appropriate piecewise variation of the displacements across the thickness (zig-zag models). No details are here reported for the structural model; readers are referred to the paper by Icardi [6].

However some observations about the modelling of the transverse deformation are required: 
(i) The transverse normal stress and strain play a primary role in sandwich composites, these materials being rather thick. In fact, in many cases, their behaviour is dominated by a nearly-without-bending crushing at the impact point. This makes an accurate modelling of the transverse displacement of primary importance for describing their behaviour. For this reason, the current post-processing technique updates the strain energy due to the transverse displacement and strain.

(ii) Since the honeycomb core crushes at a non zero stress level with an elasto-plastic behaviour, an inner force has to be accounted for in the finite element model to properly describe the degradation of the out-of-plane properties of sandwich composites, as shown by Kärger et al. [7]. The crush strength and the corresponding stiffness should be determined from specific material tests, approximated from results of experiments under combined loading, or postulated. A different approach with an equivalent result is obtained by Palazotto et al. [3] simulating the core as a foundation supporting the face sheet in their local model. The core stiffness is assumed constant till the core reaches the yield stress and strain, then between the yield strain and the failure strain the stiffness is varied to maintain the core yield stress at the lower surface of the top face sheet.

\section{Energy updating procedures}

The basic features of the standard, parent finite element based on the FSDPT model are briefly summarised hereafter; the readers could find the omitted details in the standard textbooks. This parent element is applied for computing the membrane stresses used in the post-processing phase. In order to have a computationally efficient $\mathrm{C}^{0}$ finite element, define as nodal d.o.f. the rotations of the normals $\theta_{\mathrm{x}}, \theta_{\mathrm{y}}$. The shape function matrix includes von Karman nonlinear contribution; this geometric nonlinearity is considered, because at the impact point the transverse displacement can be quite large, even when the plate deflection is small. This element would suffer from shear locking, but the problem should be overcome by the strain energy updating process outlined hereafter, because the true relation between bending and shear is accounted for by the zig-zag model.

With a post-processing procedure compatible with finite elements, the displacements, strains and stresses are locally interpolated around the impact point by spline functions. In this way, the stress derivatives involved by the integration of the local differential equilibrium equations are computed deriving the spline interpolation and not the shape functions. This avoids the need of unwise, high-order polynomials as element shape functions, since second order derivatives of the displacements are involved during this operation. This interpolation also makes easier the energy updating from the FSDPT model to the 3D zig-zag model. The readers could find all the details here omitted about spline interpolation in the standard textbooks. Note that the post-processing operations outlined hereafter are more cost-effective than using discrete-layer models, because post-processing and energy updating are carried out only locally 
around the impact point and performed on the spline interpolation, not on the finite element model.

Firstly, the FSDP model is rearranged, to be consistent with the representation of the zig-zag model. Assume $\mathrm{u}^{(\mathrm{o})}, \mathrm{v}^{(\mathrm{o})}, \mathrm{w}^{(\mathrm{o})}, \gamma_{x}$ and $\gamma_{y}$ be the functional d.o.f. of the FSDPT model, while the homologous terms of the zig-zag model be indicated by the symbol $\sim$. The functional d.o.f. of the zig-zag model be expressed as a sum of those of the FSDPT model and corrective terms:

$$
\begin{aligned}
& \tilde{u}^{(0)}=\hat{u}^{(0)}+\Delta \hat{u}^{(0)}, \quad \tilde{v}^{(0)}=\hat{v}^{(0)}+\Delta \hat{v}^{(0)}, \quad \tilde{w}^{(0)}=\hat{w}^{(0)}+\Delta \hat{w}^{(0)}, \\
& \tilde{\gamma}_{x}^{(0)}=\hat{\gamma}_{x}^{(0)}+\Delta \hat{\gamma}_{x}{ }^{(0)}, \quad \tilde{\gamma}_{y}^{(0)}=\hat{\gamma}_{y}{ }^{(0)}+\Delta \hat{\gamma}_{y}{ }^{(0)} .
\end{aligned}
$$

It is worthwhile to remark that the d.o.f. of the FSDPT model are those computed from the finite element model (i.e., the parent element) and after interpolated around the impact point by spline functions, while the corrective terms are unknown. The transverse shear strains $\varepsilon_{\mathrm{xz}}, \varepsilon_{\mathrm{yz}}$ are made least square compatible with their consistent counterparts.

The corrective terms are introduced one at a time in the compatibility condition, reiterating till convergence and solving by the penalty function method. At the first iteration, the continuity functions and the displacements are those corresponding to $f^{2}=0$; once the displacement corrections for the zig-zag model are known, the new continuity functions are computed and used as entry values for the next iteration. This procedure always converges after very few iterations, since very small corrections have been required with respect to the starting case.

As a second step, the updating of the transverse shear energy is performed equating the homologous quantities of the FSDPT and zig-zag models:

$$
\left(q_{e}+\Delta q_{e K}\right)^{T} K_{f s d p t}\left(q_{e}+\Delta q_{e K}\right)=q_{e}^{T} K_{z i g-z a g} q_{e} .
$$

where $\boldsymbol{q}_{e}$ represents the vector of nodal d.o.f., $\Delta \boldsymbol{q}_{\boldsymbol{e}}$ the corrective terms to this vector and $\boldsymbol{K}$ is the stiffness matrix (only the rows and columns relative to the out-of-plane shears). Again the solution is found by the penalty function method minimising the difference of the two members, introducing the corrections to the nodal d.o.f. one at a time and reiterating till convergence.

An approximate expression of the transverse normal stress ${ }^{\circ} \sigma_{z z}$ is obtained integrating the spline interpolation of the transverse shear stresses by the third of local differential equilibrium equations. Then an approximate expression of the transverse normal strain ${ }^{\circ} \varepsilon_{\mathrm{zz}}$ is obtained by the $3 \mathrm{D}$ stress-strain relation of elasticity using the stresses calculated at the current stage. An improved transverse normal stress ${ }^{\mathrm{o}^{*}} \sigma_{\mathrm{zz}}$ is computed substituting the expression of ${ }^{\circ} \varepsilon_{\mathrm{zz}}$ into the stress-strain relations of linear elasticity. The transverse normal stress ${ }^{{ }^{*}} \sigma_{\mathrm{zz}}$ is then used to compute improved transverse shear stresses $\sigma_{\mathrm{xz}}, \sigma_{\mathrm{yz}}$ computing one of them at a time by the third differential equilibrium equation, repeating till convergence. Once the improved stresses $\sigma_{\mathrm{xz}}, \sigma_{\mathrm{yz}}, \sigma_{\mathrm{zz}}$ are computed, they are spline interpolated for the subsequent operations. 
About the membrane energy, the respective displacements are expected to not vary so much. However, the displacements that have been improved by the transverse shear energy updating are now used to improve the membrane energy.

Finally, the improved displacements resulting from the updating process outlined above are used for the dynamics of the FSDPT models. For this purpose, $\boldsymbol{q}_{\boldsymbol{e}}$ is the current converged vector of nodal d.o.f. and the second order time derivatives are expressed as the product of spatial functions and an arbitrary function of time $\left(F_{f}(\mathrm{t})\right.$ in the FSDPT model and as $F_{z z}(\mathrm{t})$ in the zig-zag model). The displacement correction $\Delta \mathbf{q}_{\mathrm{eM}}$ that makes the work of inertial forces of the FSDPT models consistent with the one of the zig-zag model is computed by:

$$
\left(q_{e}+\Delta q_{e M}\right)^{T} M_{f s d p t}\left(q_{e}+\Delta q_{e M}\right)^{T} F_{f}(t)=q_{e}{ }^{T} M_{z i g-z a g} q_{e}{ }^{T} F_{z z}(t) .
$$

where $\boldsymbol{M}_{f s d p t}$ and $\boldsymbol{M}_{z i g-z a g}$ are the consistent mass matrices of the two involved models. Also in this case, the solution is found by the penalty function method introducing the corrections one at a time and reiterating till convergence.

\section{Simulation of the impact}

While a foreign object impacts on a structure, a locally distributed, time-varying force is induced. Since the local stresses, deformations and motion influence the contact area, the impact is a non-conservative, non-linear problem that requires a simultaneous solution of contact and dynamic equations. The Newmark implicit time integration scheme is here used, because the explicit time integration schemes require extremely small time steps in order to be stable. While in the former impact studies [8-10] the structure was described with the weighted residual method, here the finite element model, the related strain energy updating and the described post-processing techniques are used for computing the contact force, in order to accurately describe the core crushing. The modelling of the contact force is that proposed by Palazotto et al. [3].

Assume that force $F$ and the vertical displacement are zero at the first instant of the contact. After a small time interval $\Delta t$, in which no damage is though to occur, assume the contact force to reach the value $F=\Delta F$ and be distributed over the contact area according to the Hertzian law. As a consequence of the applied load the impactor moves over a distance, which depends on the nonlinear effective plate stiffness (including the plate stiffness and the contact stiffness). According to Palazotto et al. [3], the contact radius is computed within each load step by an iterative algorithm which forces the impacted top surface to conform, in the least-squares sense, to the shape of the impactor, i.e. to a sphere, applying appropriate displacements. The iterations are repeated till the impactor and the indentation radii are in agreement, then the failure analysis is performed and the local properties reduced where failure occurred. The load is then incremented and the process repeated. Since very few iterations are required for calculating the contact radius at any time step, this technique accurately describe the contact force and its distribution over the (variable) contact area does not require a much larger effort than distributing the contact force over a fixed surface as customary. 


\section{Damage criteria and post-failure degradation model}

Macromechanical, stress-based criteria with damage evolution appear to date the only affordable failure models for impact studies, having the accuracy required for design purposes with a low computational effort. At low energies, sandwich composites fail by invisible core crushing under combined shear and compression stresses, as it appears, e.g., by the experimental work of Besant et al. [11]; thus a model of this core material degradation has to be implemented in the computer codes. Since the laminated faces can behave quite differently with respect to the metallic faces considered by Besant et al., also the criteria for matrix cracking, fibres failure and delamination are implemented in the present paper. Likewise in the former paper [10], where the readers find the details here omitted, the latest 3D version of the Hashin's criterion with in situ strengths is used to predict the failure of fibres and matrix. The Choi-Chang's delamination criterion and a heuristic delamination criterion, that also considers the contribution of the transverse interlaminar stress $\sigma_{33}$, are considered. The degradation of the properties due to the accumulation of damage, according to the former failure modes, is simulated using the ply-discount theory.

However, to properly describe the core damage, the elasto-plastic behaviour of the core have to be accounted for, as shown in [7] and above reminded. The stress analysis being carried out over the grid of the spline interpolation, the failure occurring at any grid points is assumed to occur in the sub-element square region centred over the grid.

\section{Numerical results and discussion}

As an assessment (Sample case \#1) of the contact model, here the case of a square sandwich panel with a high-density foam core is considered. The side dimension is $76.2 \mathrm{~mm}$, the overall thickness is $15.863 \mathrm{~mm}$, the high-density foam core is $12.7 \mathrm{~mm}$-thick. To be self-contained, for all the omitted details, the readers are referred to Anderson [12]. The thin face sheets are made of LTM45EL-CF011-WF plies staked with a $\left[0^{\circ} / 90^{\circ} / 0^{\circ}\right]$ sequence. The panel is impacted by a steel sphere, with a mass of $1.8 \mathrm{~kg}$, a diameter of $25.4 \mathrm{~mm}$ and an impact velocity of $3 \mathrm{~m} / \mathrm{s}$. The contact force time history predicted for this case by the present model is confronted in Figure 1 with the one measured during the experiment by Anderson, while his results by a SDOF model are omitted because inaccurate. The present model appears able to accurately describe the contact problem with low computational efforts (run time of $140 \mathrm{sec}$. on a single CPU personal computer for a mesh with 100 elements, refined around the impact point) except just before the force peak.

As a second case (Sample case \#2), consider the experiments by Schubel et al. [13]. Sandwich panels with closed-cell PVC foam core (Divinycell H250) and laminated carbon fabric/epoxy faces (AGP370-5H/3501-6S) have been impacted with energies ranging from 7.75 to $108 \mathrm{~J}$. The sandwich panels have been fabricated by bonding the face sheets to the foam core with Hysol 9430 adhesive 


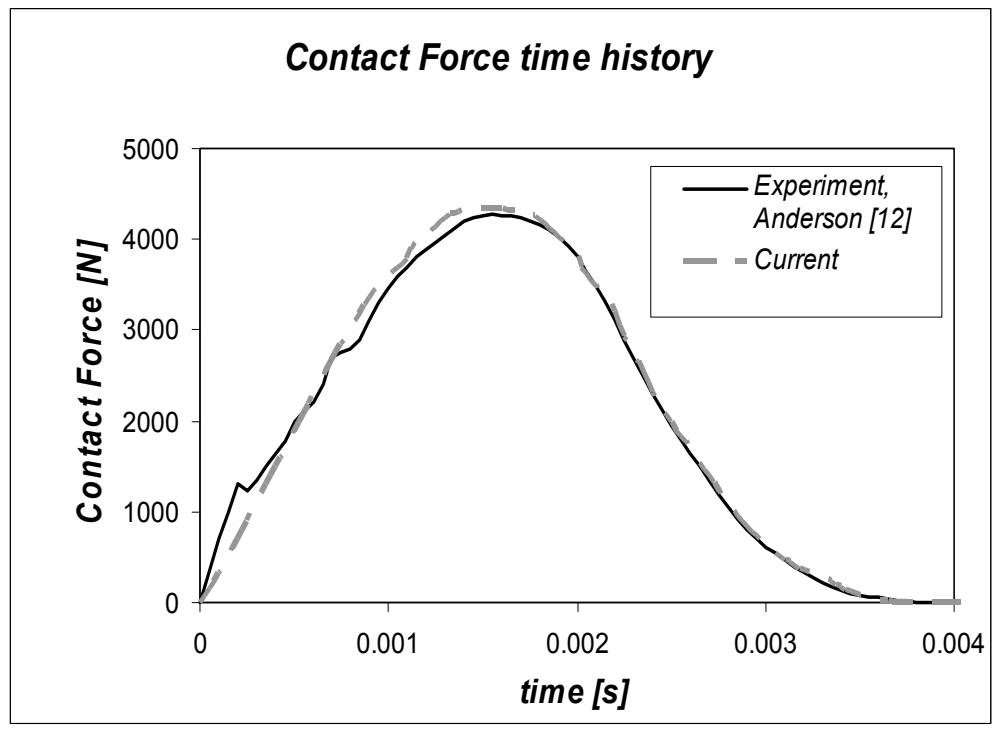

Figure 1: $\quad$ Sandwich with foam core (Sample case \#1).
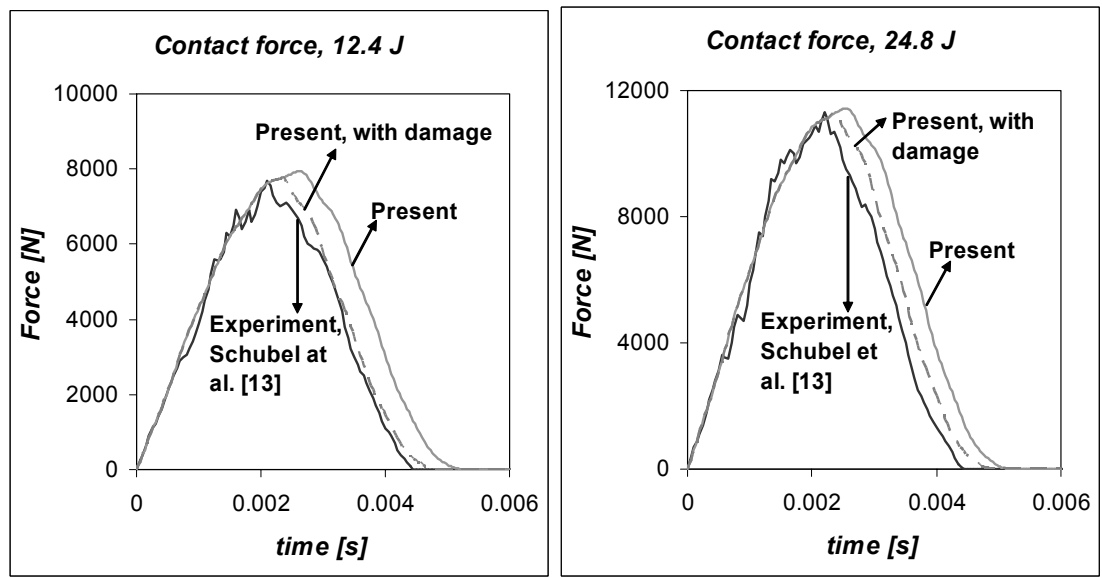

Figure 2: $\quad$ Sandwich with foam (Sample case \#2).

epoxy resin. For all the omitted details, the readers are referred to [13]. A drop tower apparatus with a free falling mass has been used to impacts the plates. The impactor was spherical, with a radius of $12.7 \mathrm{~cm}$ and a mass of $6.22 \mathrm{~kg}$. Increasing the heights of mass drop, the above mentioned impact energies have been obtained, with velocities ranging from 1.6 to $5 \mathrm{~m} / \mathrm{s}$. The latter case corresponds to an induced visible damage. The contact force time history has been computed by the present model for the energy levels of 12.4 and $24.8 \mathrm{~J}$ (i.e. low and intermediate); the comparison with the impact load measured during the 
experiments is reported in Figure 2. The peak of the contact load is overestimated at the lowest energy level, while at the higher ones it is quite accurately predicted. The failure models employed appears unable to accurately predict the first damage threshold appearing just before the load peak. This either means that the failure modes related with these points cannot be described in terms of fibres and matrix failures, delamination and core crushing, that such failure models does not represent the effective levels of failure, or that the contact force model and the time integration procedure are not good enough. It is believed that to obviate this drawback, a micro-mechanics failure analysis should be carried out, which is beyond the purpose of the present computational model.

Consider now the sample case formerly studied by Kärger et al. [7] (Sample case \#3), for which experimental results are available for the contact force and for the damage. A rather sophisticate computational model based on a three-layer description for the stress and strain field across the sandwich thickness is used in [7]; the core damage is represented using the criterion of Besant et al. [11] and the elasto-plastic crushing of the core considering the contribution to the work of the inner force, degrading the out-of-plane properties of the core after failure. No failure of the face sheets have been accounted for, although the authors observed that the face sheet degradation could provide a more accurate contact force time history prediction. The contact force has been computed using the Hertzian law and the Newmark time integration scheme; this load has been parabolically distributed over the contact area and the analysis has been carried out on a quarter of the plate, supposing the problem symmetric.

The sandwich panels are made of 28 mm-thick NOMEX honeycomb 4.8-48, a very thin top face sheet $(0.633 \mathrm{~mm})$ of Cytec $977-2 / \mathrm{HTA}$ material and a thick bottom face sheet $(2.7 \mathrm{~mm})$ made of CFRP fabric plies and UD tapes and they

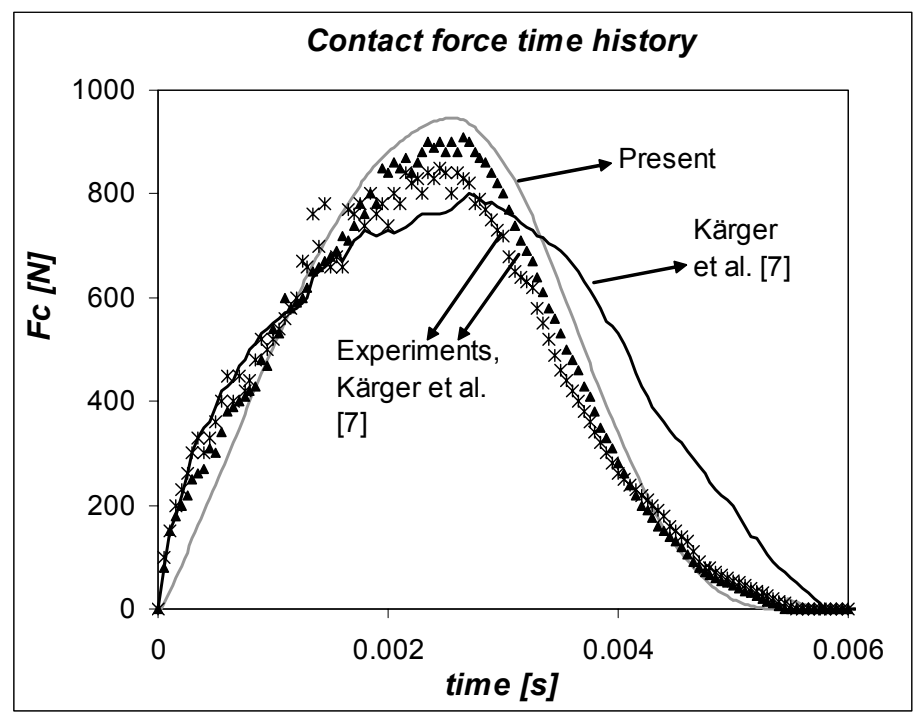

Figure 3: $\quad$ Sandwich with foam (Sample case \#3), contact force. 
have been impacted with energies of 1 and $8 \mathrm{~J}$. For each energy level, two experimental force time histories have been presented to display the scatter of results. The panels have been completely supported at their bottom face sheet and impacted by a steel sphere with a diameter of $25.4 \mathrm{~mm}$ and a mass of 1.10 $\mathrm{kg}$. The contact force histories at $1 \mathrm{~J}$ as predicted by the model of Kärger et al., by the present computational model and measured at ILR Dresden are reported in Figure 3. The damage detected via ultrasonic inspection at ILR is depicted in Figure 4 (our digital contour elaboration) and compared to the one computed by the present method. The residual stiffness after core crushing has been computed reducing the out-of-plane properties of the core by the use of the average factors suggested in [7], starting from a contact force of $0.23 \mathrm{kN}$, correspondent to the onset of core damage.

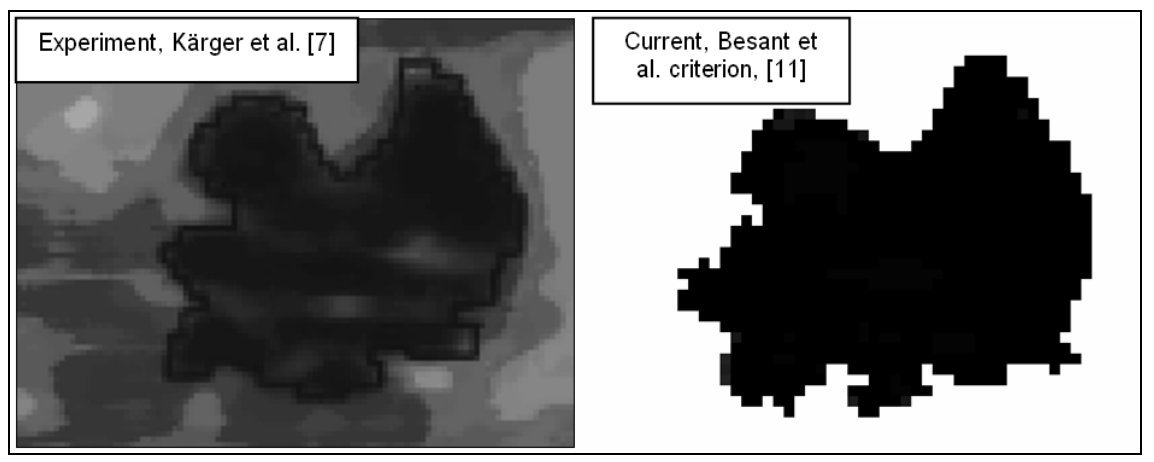

Figure 4: $\quad$ Sandwich with foam (Sample case \#3), damage analysis.

\section{Concluding remarks}

The impact force time history and the impact induced damage of sandwich composites with laminated faces and foam or honeycomb core have been presented. The comparison with the experimental results available in literature for these sample cases shows the current numerical model to be quite accurate and suitable for improving the predictions of the conventional structural models by updating their strain energy, as illustrated. The failure and post-failure models used, even if simple stress based criteria, appear accurate enough for predicting either the core crushing at low energy levels, or the face sheet failure at higher energy levels.

\section{References}

[1] Lin, C., Hoo Fatt, M.S., Perforation of composite plates and sandwich panels, J. of Composite Materials, 40(20), pp. 1801-1839, 2006.

[2] Suvorov, A.P., Dvorak, G.J., Enhancement of low velocity impact damage resistance of sandwich plates, Int. J. Solids Struct., 42, pp. 2323-2344, 2005. 
[3] Palazotto, A.N., Herup, E.J., Gummadi, L.N.B., Finite element analysis of low-velocity impact on composite sandwich plates, Compos. Struct., 49, pp. 209-227, 2000.

[4] Icardi, U., A Three-dimensional zig-zag theory for analysis of thick laminated beams, Compos. Struct., 52, pp. 123-135, 2001.

[5] Icardi, U., Higher-order zig-zag model for analysis of thick composite beams with including transverse normal stress and sublaminates approximations, Composites-Part B, 32b, pp. 343-354, 2001.

[6] Icardi, U., Applications of zig-zag theories to sandwich beams, Mech. of Adv. Mat. Struct., 10(1), pp. 77-97, 2002.

[7] Kärger, L., Baaran, J., Teßmer, J., Rapid simulation of impacts on composite sandwich panels inducing barely visible damage, Compos. Struct., 79, pp. 527-534, 2007.

[8] Icardi, U., Zardo, G., $\mathrm{C}^{\circ}$ plate element for delamination damage analysis based on a zig/zag model and strain energy updating, Int. J. of Impact Engineering, 31(5), pp. 579-606, 2005.

[9] Icardi, U., $C^{\circ}$ Plate element for global/local analysis of multilayered composites, based on a 3D zig-zag model and strain energy updating, Int. J. of Mechanical Sciences, 47, pp. 1561-1594, 2005.

[10] Icardi, U., C ${ }^{\circ}$ Plate Element Based on Strain Energy Updating and Spline Interpolation, For Analysis of Impact Damage in Laminated Composites, Int. J. of Impact Engineering, 34, pp. 1835-1868, 2007.

[11] Besant, T., Davies, G.A.O., Hitchings, D., Finite element modelling of low velocity impact of composite sandwich panels, Composites-Part A, 32A, pp. 1189-1196, 2001.

[12] Anderson, T.A., An investigation of SDOF models for large mass impact on sandwich composites, Composites-Part B, 36, pp. 135-142, 2005.

[13] Schubel, P.M., Luo, J.J., Daniel, I.M., Low velocity impact behavior of composite sandwich panels, Composites: Part A, 36, pp. 1389-1396, 2005. 